\title{
The Polish adaptation of the Mental Health Continuum-Short Form (MHC-SF)
}

\author{
Karaś, Dominika ; Cieciuch, Jan ; Keyes, Corey L.M.
}

\begin{abstract}
The Mental Health Continuum-Short Form (MHC-SF) developed by Keyes (2009) is the tool that allows for continuous assessment of subjective well-being (including its three aspects: emotional, psychological, and social), as well as for the categorical diagnosis of the presence of mental health and the absence of mental health (understood as flourishing and languishing). This paper presents the result of the validation and psychometric parameters of the Polish MHC-SF. The participants included 2115 respondents aged 16-81 (55.6\% women) from Poland. The findings confirmed the reliability of MHC-SF, external validity, three-dimensional structure of subjective well-being, and supported two-continua model of mental health, where mental health and mental illness are two related but distinguishable dimensions, not at the ends of the same continuum.
\end{abstract}

DOI: https://doi.org/10.1016/j.paid.2014.05.011

Posted at the Zurich Open Repository and Archive, University of Zurich ZORA URL: https://doi.org/10.5167/uzh-175140

Journal Article

Originally published at:

Karaś, Dominika; Cieciuch, Jan; Keyes, Corey L.M. (2014). The Polish adaptation of the Mental Health Continuum-Short Form (MHC-SF). Personality and Individual Differences, 69(October):104-109.

DOI: https://doi.org/10.1016/j.paid.2014.05.011 
The Polish Adaptation of the Mental Health Continuum-Short Form (MHC-SF)

\author{
Dominika Karaś \\ Cardinal Stefan Wyszyński University, Warsaw, Poland \\ Jan Cieciuch \\ University of Finance and Management, Warsaw, Poland \\ and University of Zurich, Switzerland
}

Corey L. M. Keyes

Emory University, Atlanta, USA

Correspondence concerning this paper should be addressed to:

Dominika Karaś

Institute of Psychology, Cardinal Stefan Wyszyński University, Poland

Address: ul. Wóycickiego 1/3, 01-938 Warsaw, Poland.

Phone number: +48 225699602

Fax number: +48225699607

E-mail: dominika.karas@gmail.com

\title{
Acknowledgements
}

Dominika Karaś was supported by Grants (UMO-2012/07/N/HS6/02015) from the Polish National Science Centre. Jan Cieciuch was supported by Grants (DEC2011/01/D/HS6/04077) from the Polish National Science Centre. 


\begin{abstract}
The Mental Health Continuum-Short Form (MHC-SF) developed by Keyes (2009) is the tool that allows for continuous assessment of subjective well-being (including its three aspects: emotional, psychological, and social), as well as for the categorical diagnosis of the presence of mental health and the absence of mental health (understood as flourishing and languishing). This paper presents the result of the validation and psychometric parameters of the Polish MHC-SF. The participants included 2115 respondents aged 16-81 (55.6\% women) from Poland. The findings confirmed the reliability of MHC-SF, external validity, threedimensional structure of subjective well-being, and supported two-continua model of mental health, where mental health and mental illness are two related but distinguishable dimensions, not at the ends of the same continuum.
\end{abstract}

Keywords: Mental Health Continuum, subjective well-being, emotional well-being, psychological well-being, social well-being 


\section{The Polish Adaptation of the Mental Health Continuum-Short Form (MHC-SF)}

Until recently, mental health has been understood as the absence of mental illness. But in last few decades, this view has changed considerably. The current definition of mental health, created by WHO (2004, p.12) states: "Mental health is a state of well-being in which every individual realizes his or her own potential, can cope with the normal stresses of life, can work productively and fruitfully, and is able to make a contribution to her or his community." This definition is consistent with a general definition of health, which describes health as a state consisting of the presence of well-being and not only the absence of illness or disability (WHO, 1948).

When mental health is defined positively, well-being is an important component. Subjective well-being (SWB) - individuals reports of their own well-being - consists of two conceptual traditions, the hedonic and the eudaimonic. The first of which equates well-being with feeling good about (i.e., positive emotions toward) one's life, while the second with functioning well in life as an individual and a citizen. The hedonic tradition focuses on emotional well-being through the measurement of satisfaction with life and positive affect (Diener, 1984). The tradition of eudaimonia focuses on psychological (Ryff, 1989) and social (Keyes, 1998) well-being, both measured with multidimensional scales. The euaimonic stream assesses how well individuals see themselves functioning in life against secular standards of excellence such as purpose, contribution, integration, autonomy, intimacy, acceptance, and mastery. Studies support the tripartite structure of SWB, consisting of emotional, psychological, and social well-being in U.S. (see e.g., Gallagher, Lopez \& Preacher, 2009; Robitschek \& Keyes, 2009) and increasingly across cultures (Keyes, 2013). The Mental Health Continuum "short form" (MHC-SF) was created to address the problem of the diagnostic threshold and to create a version more efficiently administered in epidemiological surveillance. The MHC-SF derives from the long form (MHC-LF) used in 
the Midlife in the United States (MIDUS) study (Keyes, 2002). While the MHC-LF consisted of 40 items, the MHC-SF consists of 14 items representing the construct definition for each facet of well-being. Three items indicate emotional well-being, six items represent the six dimensions of psychological well-being, and five items represent the five dimensions of social well-being. The response option for the short form was changed to measure the frequency (from "never" to "every day") with which respondents experienced each sign of mental health during the past month.

Keyes (2002) argues that good mental health is a syndrome of feeling good and functioning well. In the same way that depression requires symptoms of an-hedonia, mental health consists of symptoms of hedonia. But, feeling good, in the same way as only feeling sad or losing interest in life, is not sufficient for the diagnosis of a clinical state. Rather, and in the same way that major depression consists of symptoms of mal-functioning, mental health must also consist of symptoms of positive functioning.

In turn, the mental health continuum (Keyes, 2002) consists of three levels of positive mental health: flourishing, moderate, and languishing mental health (although researcher can use the "flourishing" and "not flourishing" categorization). Individuals with flourishing mental health report feeling at least one measure of hedonic well-being plus six or more of the measures of positive functioning almost every day or every day during the past month. Individuals with languishing mental health, however, report feeling at least one measure of hedonic well-being with six or more measures of positive functioning never or maybe once or twice during the past month. Languishing is the absence of mental health - a state of being mentally $\boldsymbol{u n h e a l t h y}$ - which is tantamount to being stuck and stagnant, or feeling empty or that life lacks interest and engagement. Individuals who are neither flourishing nor languishing are diagnosed with moderate mental health. 
The short form of the MHC has shown excellent internal consistency $(>.80)$ and discriminant validity in adolescents and adults in the U.S., but increasingly cross-culturally (Keyes, 2013). Lamers et al (2011) evaluated the measurement invariance of the MHC-SF using data from a large representative sample of Dutch adults. This study used item response analysis to examine differential item functioning (DIF) by seven demographic variables (e.g., age, education, sex), health (i.e., whether a respondent had any of 18 disease conditions or not), and over time (i.e., respondents were followed up 4 times over a 9 month period). Of the 14 items in the MHC-SF, none showed DIF over time or health status, and only 4 showed DIF by demographics ( 1 social well-being item by sex and one by education, and 2 psychological well-being item by age group). Adjustment for DIF on the 4 items did not affect the results of mean comparison, indicating that the DIF was small on those 4 items. Overall, the MHC-SF items have the same meaning by demographics, health, and over time.

Similarly, Joshanloo and colleagues (2013) found support for the factor structure (emotional, psychological, and social) and full metric invariance of the MHC-SF across three cultures: Netherlands, South Africa and Iran. Metric invariance means that the strength of the relationships between the MHC-SF items and latent factors are equivalent across the populations. As such, comparisons of MHC-SF scores across these three cultures reflect grop differences rather than differential item meaning by culture. In addition, the long and short forms of the MHC have been useful in improving the scientific understanding of the risk of future mental illness (MDE, GAD, and panic attacks; see Keyes, Dhingra, \& Simoes, 2010), risk of premature mortality (Keyes \& Simoes, 2012), healthcare utilization, missed days of work, disability as measured by limitations of ADL (Keyes, 2007), resilience (Keyes, 2009), and self reported academic impairment among colleges students (Keyes, Eisenberg, Perry, Dube, Kroenke, \& Dhingra, 2012). 
The research presented in this article is the first attempt to validate the MHC-SF one in the context of Eastern Europe. To that end, we investigate the psychometric properties of the Polish adaptation of MHC-SF, focusing on the following: 1) internal alpha consistency; 2) structural validity assessed via confirmatory factor analysis; 3) measurement invariance across gender and educational groups assessed in multi-group confirmatory factor analysis; 4) external validity, assessed by inspection of correlation with different measures of some aspects of well-being; and 5) validity of the two-continua model assessed by factor analysis of well-being and mental illness measures.

\section{Methods}

\section{Participants}

The participants included 2115 (55.6\% females) adults from Poland, aged 16-81 $(M=$ $28.89, S D=10.62$ ). They completed the study measures as an anonymous self-report questionnaire.

The data were collected in 2013 in four studies in four different samples. In Study 1, there were 655 participants $(49.8 \%$ females) aged $25-60(M=37.16, S D=5.22), 0.3 \%$ with primary education, $4.7 \%$ with vocational education, $23 \%$ with secondary education, and $35 \%$ with higher education. In Study 2, 835 respondents participated (56.1\% females) aged 16-81 $(M=29.56, S D=12.281), 5 \%$ with primary education, $4 \%$ with vocational education, $55.6 \%$ with secondary education and 27.6\% with higher education. In Study 3, 477 participants took part $(63.3 \%$ females $)$ aged $18-33(M=21.51, S D=1.961)$. All of them were students. In Study 4, there were 148 participants attending high school (53.4\% females) aged $16-19(M=$ $17.24, S D=.788)$

\section{Measures:}

Mental Health Continuum-Short Form (MHC-SF, Keyes, 2013). The MHC-SF consisted of 14 items that represent various facets of well-being (the items were chosen from 
the longer version of this tool, as the most prototypical for each facet of well-being). The answering scale is 6-points, and describes the frequency of experiencing various symptoms of well-being. The scale ranges from never to everyday (during the past month).

This questionnaire allows for two kinds of assessment. On one hand, with MHC-SF we can assess the level of well-being (and its three dimensions: social, psychological, and emotional). On the other, this tool also allows for categorization into three types of mental health: flourishing, languishing, and moderate mental health.

To be diagnosed with flourishing, one should answer everyday or almost everyday (during past month) at least once in the emotional well-being scale, and at least six times across eleven items measuring social and psychological well-being. To be diagnosed with languishing, one has to experience never or once or twice during the past month for at least one item from the emotional well-being scale and at least six items on the psychological functioning (social and psychological well-being) scales. The respondents classified neither as flourishing nor as languishing are moderately mentally healthy.

General Health Questionnaire (GHQ-28). The Polish version of GHQ-28 (Goldberg \& Hillier, 1979; Polish adaptation: Makowska \& Merecz, 2001) is the questionnaire, where the respondent is asked to assess his or her health frequency of experiencing various psychical and physical states during the last couple of weeks. The main objective of this questionnaire is for measuring various mental disorders.

The questionnaire has 28 items comprising four scales: somatic symptoms, anxiety and insomnia, social dysfunction and severe depression. The responding scales range from not at all to much more than usual, better than usual to much worse than usual, and definitely no to definitely yes. The GHQ-28 was used in Study 2 and its reliability assessed with Cronbach's alpha which ranged from .793 (Somatic Symptoms) to .880 (Severe Depression). 
Running head: POLISH ADAPTATION OF MENTAL HEALTH CONTINUUM-SHORT FORM

Positive and Negative Affect Schedule - Expanded Form (PANAS-X). The Polish version of PANAS-X (Watson \& Clark, 1994; Polish adaptation: Fajkowska \& MarszałWiśniewska, 2009) includes 60 items measuring positive and negative affect and is composed of 12 subscales (presented in Table 4) using a 5-point Likert scale, ranging from 1 (not at all) to 5 (extremely). A respondent has to assess the intensity of experiencing various feelings and emotions. The PANAS-X was used in Study 2 and its reliability ranged from .670 (Serenity) to .896 (Fatigue).

The Questionnaire for Eudaimonic Well-Being (QEWB). Eudaimonic Well-Being, according to Waterman et al. (2010), is the active development of a human's best potential and perceiving them as personally expressive. The QEWB (Waterman et al., 2010; Polish adaptation by Kłym, Karaś, Najderska, \& Cieciuch, 2014) is a measurement tool composed of 21 items with a Likert-type response scale ranging from 1 (completely agree) to 7 (completely disagree) and it was designed to assess the general level of eudaimonic well-being. The QEWB was used in Study 3 and its reliability assessed with Cronbach's alpha was .870.

\section{Results}

\section{Reliability of MHC-SF}

The Cronbach's alpha coefficients for each scale and the total score of MHC-SF are presented in Table 1. All of the coefficients can be considered satisfactory.

Table 1

Cronbach's $\alpha$ coefficients of Mental Health Continuum-Short Form

Study 1 Study 2 Study 3 Study 4 Total Sample

$\begin{array}{llllll}\text { Emotional well-being } & .86 & .86 & .84 & .71 & .85 \\ \text { Social well-being } & .88 & .81 & .78 & .72 & .82\end{array}$


Running head: POLISH ADAPTATION OF MENTAL HEALTH CONTINUUM-SHORT FORM

$\begin{array}{lccccc}\text { Psychological well-being } & .90 & .87 & .85 & .79 & .87 \\ \text { Total score } & .94 & .91 & .90 & .84 & .91\end{array}$

\section{The Structure of Well-Being}

Confirmatory Factor Analysis. Since the three-dimensional structure of well-being was confirmed in previous research (cf. Keyes et al., 2008; Lamers et al., 2011), in this study we proceeded using only confirmatory factor analysis on items in each sample separately, as well as in the whole sample. Model fit coefficients are presented in Table 2. Reported CFI coefficients (all above .900) and almost all SRMR coefficients (only in one sample SRMR was higher than .08) allow for accepting the three dimensional model in all samples based on the commonly used criteria (Hu \& Bentler, 1999; Marsh, Hau, \& Wen, 2004). Only the RMSEA parameter was slightly above the cut-off in most samples.

Table 2

Model fit coefficients from confirmatory factor analysis in each sample (df=74)

\begin{tabular}{lllll}
\hline & $\chi^{2}$ & CFI & RMSEA & SRMR \\
Study 1 & 564.290 & .933 & .093 & .058 \\
Study 2 & 645.159 & .921 & .090 & .059 \\
Study 3 & 385.918 & .926 & .081 & .059 \\
Study 4 & 160.194 & .912 & .073 & .085 \\
Total Sample & 1454.358 & .932 & .084 & .054 \\
\hline
\end{tabular}

Note. $\chi^{2}=$ Chi-Square; $d f=$ degrees of freedom; CFI = Comparative Fit Index; RMSEA = Root Mean Square Error of Approximation; SRMR = Standardized Root Mean Square Residual. 
Figure 1 presents the results of the confirmatory factor analysis from the whole sample: correlations between each well-being dimension and factor loadings of items used in MHC-SF. All presented correlations are significant with $p<.001$.

The results confirm that the three-dimensional structure including emotional, psychological, and social well-being is sufficiently fitted to the data in all examined groups.

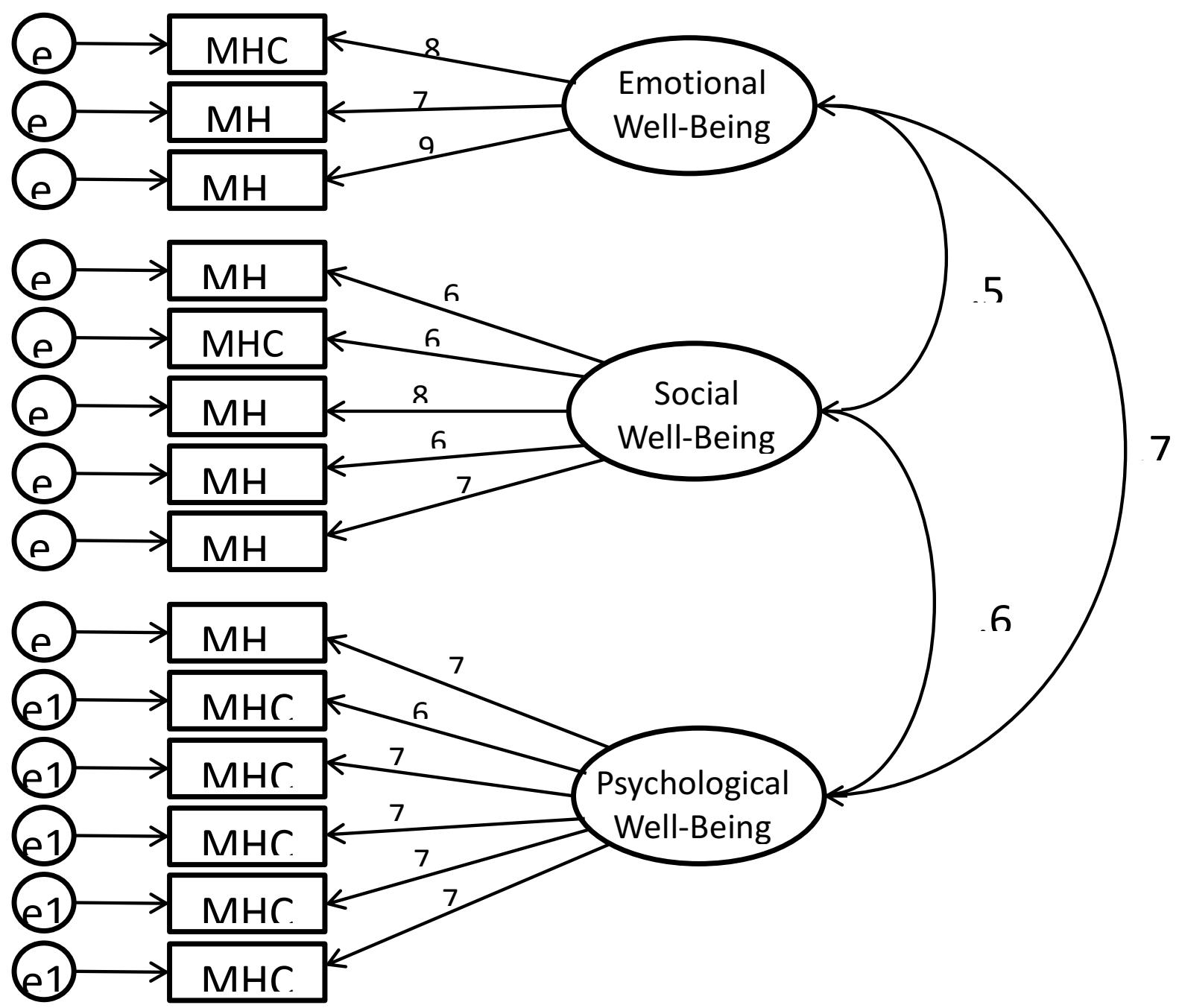

Figure 1. Confirmatory Factor Analysis of Well-Being Three-Dimensional Structure.

Measurement Invariance. The multigroup confirmatory factor analysis was performed to test measurement invariance of well-being measures across gender and 
educational groups for the whole sample. We distinguished five educational groups: people with primary or vocational education, people with secondary education, people with higher education (those three groups came from Study 1 and Study 2), high school pupils (Study 4), and university students (Study 3). The results are presented in Table 3. Using the common rules proposed by Chen (2007), full configural, full metric, and full scalar invariance were supported for both gender and educational groups.

Table 3

Measurement invariance of three dimensions of the MHC-SF across gender and educational groups

\begin{tabular}{|c|c|c|c|c|}
\hline & \multicolumn{4}{|c|}{ Model fit indices } \\
\hline & $\chi^{2}$ & $d f$ & CFI & RSMEA [90\% CI] \\
\hline \multicolumn{5}{|l|}{ Educational groups } \\
\hline Configural invariance & 1336.533 & 350 & .927 & $.039[.037, .042]$ \\
\hline Metric invariance & 1390.908 & 394 & .926 & $.037[.035, .039]$ \\
\hline Scalar invariance & 1556.600 & 438 & .917 & $037[.035, .039]$ \\
\hline \multicolumn{5}{|l|}{ Gender groups } \\
\hline Configural invariance & 1328.206 & 142 & .928 & $.060[.057, .064]$ \\
\hline Metric invariance & 1250.938 & 153 & .928 & $.058[.055, .061]$ \\
\hline Scalar invariance & 1357.985 & 164 & .922 & $.059[.056, .062]$ \\
\hline
\end{tabular}

Note. $\chi^{2}=$ Chi-Square; $d f=$ degrees of freedom; CFI = Comparative Fit Index; RMSEA $=$ Root Mean Square Error of Approximation and 90\% Confidence Interval; $\Delta=$ Change.

\section{External Validity}

To assess the external validity of MHC-SF, we verified the correlation between the scales of MHC-SF and other measurement instruments: GHQ-28, PANAS-X, and QEWB. 
Running head: POLISH ADAPTATION OF MENTAL HEALTH CONTINUUM-SHORT FORM

We expected significant correlations between MHC-SF scales and validation questionnaires (negative with GHQ-28 scales and PANAS-X negative affect scales, and positive with QEWB score). Table 4 presents the results from those analyses.

Table 4

Bivariate correlations of validation measurements and MHC-SF scales

\begin{tabular}{|c|c|c|c|c|}
\hline & $\begin{array}{l}\text { Emotional } \\
\text { Well-Being }\end{array}$ & $\begin{array}{c}\text { Social } \\
\text { Well-Being }\end{array}$ & $\begin{array}{c}\text { Psychological } \\
\text { Well-Being }\end{array}$ & $\begin{array}{l}\text { MHC-SF } \\
\text { total score }\end{array}$ \\
\hline \multicolumn{5}{|l|}{ GHQ-28 $(N=835)$ : } \\
\hline Somatic symptoms & -.31 & -.15 & -.27 & -.27 \\
\hline Anxiety/insomnia & -.42 & -.22 & -.37 & -.38 \\
\hline Social dysfunction & -.41 & -.22 & -.36 & -.37 \\
\hline Severe depression & -.45 & -.25 & -.41 & -.42 \\
\hline Total score & -.50 & -.26 & -.44 & -.45 \\
\hline \multicolumn{5}{|l|}{ PANAS-X $(N=835)$ : } \\
\hline Negative affect & -.35 & -.22 & -.36 & -.36 \\
\hline Fear & -.35 & -.20 & -.37 & -.35 \\
\hline Sadness & -.48 & -.28 & -.45 & -.46 \\
\hline Guilt & -.35 & -.19 & -.40 & -.36 \\
\hline Hostility & -.31 & -.23 & -.26 & -.30 \\
\hline Shyness & -.26 & -.15 & -.32 & -.29 \\
\hline Fatigue & -.29 & -.23 & -.30 & -.32 \\
\hline Positive affect & .42 & .31 & .46 & .46 \\
\hline
\end{tabular}




\begin{tabular}{lcccc} 
Jovialty & .53 & .37 & .46 & .51 \\
Self assurance & .37 & .29 & .49 & .45 \\
Attentiveness & .26 & .18 & .35 & .31 \\
Serenity & .42 & .22 & .35 & .37 \\
\hline QEWB $(N=477):$ & .48 & .41 & .58 & .57 \\
\hline
\end{tabular}

All correlations are significant with $p<.01$.

Note. MHC-SF $=$ Mental Health Continuum - Short Form; GHQ-28 = General Health Questionnaire, 28 items; PANAS-X = Positive and Negative Affect Schedule - Expanded Form; QEWB = the Questionnaire for Eudaimonic Well-Being.

The results confirmed the external validity of the Polish adaptation of MHC-SF. All of the scales from MHC-SF correlate negatively with the scales from GHQ-28 (the highest correlations were reported for emotional well-being, the lowest for social well-being). Also, all the scales of PANAS-X correlated significantly with the scales of MHC-SF (all correlations with negative affect symptoms were negative and positive with positive affect symptoms). For the total score of the QEWB, the highest was the correlation with psychological well-being, the lowest with social well-being.

Demographic Variables and Well-Being. Keyes and Waterman (2003) also indicated such potential determinants of well-being as: age, gender, education, personality, identity, marital status, relationships, social roles, religion, etc. The analyses in each study revealed the significant correlation of age and with all three of the well-being dimensions. For each dimension, this correlation was negative (although the correlations were weak: from -.05 to .13). We found no significant differences between men and women on any well-being dimensions.

To verify the differences in subjective well-being between people with primary, secondary and higher education, students, and high school pupils, we performed a General 
Linear Model with post hoc Tukey's test. The results revealed that there are significant differences in the total MHC-SF score and only between people with secondary education and students (students reported a higher level of subjective well-being).

\section{The Verification of Two-Continua Model}

To confirm the hypothesis that mental health and mental illness are not two sides of the same continuum, but distinguishable (however related) factors, we performed two kinds of factor analyses on MHC-SF and GHQ scales. The first one was an exploratory factor analysis with two-factor Varimax Rotation of the MHC-SF and GHQ-28 subscales. The second approach was a comparison of the three models using confirmatory factor analysis.

In the exploratory factor analysis, we expected that the scales of GHQ-28 (negative symptoms) would load onto one factor, and the scales of MHC-SF (as the indicators of wellbeing) onto a second one. The results can be seen in Table 5. This analysis revealed two separate factors: one with all of the subscales from MHC-SF, and the second with all of the subscales from GHQ-28. Those two factors explained $68 \%$ of the variance.

Table 5

Exploratory Factor Analysis with Two Factors: Mental Health and Mental Illness

Factor 1

Factor 2

Subscale

Mental Health

Mental Illness

MHC-SF:

Emotional well-being

Social well-being

Psychological well-being

.81 
GHQ-28

Somatic symptoms

Anxiety/Insomnia

Social dysfunction

Severe depression

Note. MHC-SF $=$ Mental Health Continuum - Short Form; GHQ-28 = General Health Questionnaire, 28 items. In the table, only loadings $>.40$ are presented.

In the series of three confirmatory factor analyses as expected, the two-factor model (considering two related continua) fit the data better than the other two models. The findings can be found in Table 6 . The first analysis included a model with one single factor with all of the scales from GHQ-28 and the scales from MHC-SF. This model considered the presence of mental health as the absence of mental illness. However, the fit coefficients did not confirm that a one factor model was well-fitted. The second model included two orthogonal factors (first with the scales from GHQ-28, as the indicators of mental illness and second one with the scales from MHC-SF as the indicators of mental health). Once again, the results did not support the model with two unrelated factors as well-fitted.

Eventually, the third model contained two correlated factors, in accordance with the hypothesized two-continua model. It turned out that the model with two distinct but correlated factors demonstrated much better set of fit coefficients than the other two models. This finding confirms that mental health and mental illness are distinct, but related dimensions, not being the ends of one bipolar continuum.

Table 6 
Running head: POLISH ADAPTATION OF MENTAL HEALTH CONTINUUM-SHORT FORM

Model Fit Coefficients of Three Confirmatory Factor Analyses - Verification of the Structure of Mental Health and Mental Illness

\begin{tabular}{lccccc}
\hline & $\chi^{2}$ & $d f$ & CFI & RMSEA & SRMR \\
& & & & & \\
Model 1 - Single factor & 581.028 & 14 & .751 & .220 & .106 \\
Model 2 - Two orthogonal factors & 311.360 & 14 & .870 & .159 & .218 \\
Model 3 - Two related factors & 96.026 & 13 & .964 & .087 & .050 \\
\hline
\end{tabular}

Note. $\chi^{2}=$ Chi-Square; $d f=$ degrees of freedom; CFI = Comparative Fit Index; RMSEA = Root Mean Square Error of Approximation; SRMR = Standardized Root Mean Square Residual.

The categorical diagnosis with MHC-SF enables assessing whether one is flourishing, languishing or moderately mentally healthy. The data from this study revealed that in a total sample of 2115 participants, $15.5 \%$ were languishing, $58.1 \%$ moderately mentally healthy, and $26.0 \%$ flourishing. These findings are similar to those found by Keyes et al. (2008).

\section{Discussion}

The main aim of the current study was assessing the psychometric characteristics of the Polish adaptation of MHC-SF - a questionnaire designed for measuring positive mental health. All subscales of the MHC-SF and the total scale score exhibited very good reliability. We also confirmed the tripartite structure of the MHC-SF. Moreover, our results supported the full configure invariance, metric invariance, and scalar measurement invariance of three subscales of the MHC-SF by gender and by educational attainment. Further, the results confirmed that symptoms of mental health and symptoms of mental illness do not reflect opposite ends of the same continuum, but rather load onto two separate but related factors. This means that the absence of mental diseases does not imply the presence of full mental health. And, clearly, the presence of subjective well-being does not necessarily mean that one is not suffering from mental illnesses. 
Furthermore, the external validity of the Polish version of MHC-SF was confirmed in this study, however we used only a few instruments that did not fully correspond with each MHC-SF scale, particularly with the social well-being scale. We did not find gender differences in MHC-SF total score, and found that individuals with secondary education had lower MHC-SF total score than and students.

Undoubtedly, further research on the Polish MHC-SF will be needed. For example, more research should be done to investigate the predictive validity of the MHC-SF in terms of health and illness outcomes, work productivity and sickdays from work, among other important public health outcomes. However, we believe the findings from the current study strongly suggest the reliability and construct validity of the Polish adaptation of MHC-SF.

\section{References:}

Bradburn, N. M. (1969). The structure of psychological well-being. Chicago: Aldine Publishing Company.

Chen, F. (2007). Sensitivity of goodness of fit indexes to lack of measurement invariance. Structural Equation Modeling, 14(3), 464-504.

Fajkowska, M. \& Marszał-Wiśniewska, M. (2009). Właściwości psychometryczne Skali Pozytywnego i Negatywnego Afektu - Wersja Rozszerzona (PANAS-X). Wstępne wyniki badań w polskiej próbie [The Psychometrical Parameters of Positive and Negative Affect Schedule - Expanded Form (PANAS-X). Preliminary Results from Polish Population]. Przeglad Psychologiczny [Review of Psychology], 52(4), 355-388.

Gallagher, M. W., Lopez, S. J., \& Preacher, K. J. (2009). The hierarchical structure of wellbeing. Journal of Personality, 77(4), 1025-1049. 
Running head: POLISH ADAPTATION OF MENTAL HEALTH CONTINUUM-SHORT FORM

Goldberg, D. P. \& Hillier V. F. (1979). A scaled version of the General Health Questionnaire. Psychological Medicine, 9, 191-197.

Hu, L., \& Bentler, P. M. (1999). Cutoff criteria for fit indexes in covariance structure analysis: Conventional criteria versus new alternatives. Structural Equation Modeling, $6(1), 1-55$.

Jahoda, M. (1958). Current concepts of positive mental health. New York: Basic Books.

Joshanloo, M., Wissing, M. P., Khumalo, I. P., Lamers, S. M. A. (2013). Measurement invariance of the Mental Health Continuum-Short Form (MHC-SF) across three cultural groups. Personality and Individual Differences, 55, 755-759.

Keyes, C. L. M. (1998). Social well-being. Social Psychology Quarterly, 61, 121-140.

Keyes, C. L. M. (2002). The mental health continuum: From languishing to flourishing in life. Journal of Health and Social Behavior, 43, 207-222.

Keyes, C. L. M. (2005). The subjective well-being of America's youth: Toward a comprehensive assessment. Adolescent and Family Health, 4, 3-11.

Keyes, C. L. M. (2006). Mental health in adolescence: Is America's youth flourishing? American Journal of Orthopsychiatry, 76, 395-402.

Keyes, Corey L. M. (2009). The black-white paradox in health: Flourishing in the face of inequality. Journal of Personality, 77(6), 1677-1706.

Keyes, C. L. M. (2013). Atlanta: Brief description of the mental health continuum short form (MHC-SF). Available: http://www.sociology.emory.edu/ckeyes/. [On-line, retrieved: November, 7th, 2013].

Keyes, C. L. (Ed.). (2013). Mental well-being: International contributions to the study of positive mental health. Springer. 
Running head: POLISH ADAPTATION OF MENTAL HEALTH CONTINUUM-SHORT FORM

Keyes, C. L. M., \& Lopez, S. J. (2002). Toward a science of mental health. Positive directions in diagnosis and interventions. In C. R. Snyder \& S. J. Lopez (Eds.), Handbook of positive psychology (pp. 45-59). London: Oxford University Press.

Keyes, C. L. M., \& Waterman, M. B. (2003). Dimensions of well-being and mental health in adulthood. In M. H. Bornstein, L. Davidson, C. L. M. Keyes, \& M. More (Eds.), Wellbeing. Positive development across the life course (pp. 477-497). London: Lawrence Erlbaum Associates.

Keyes, C. L. M., Wissing, M., Potgieter, J., Temane, M., Kruger, A., \& van Rooy, S. (2008). Evaluation of the Mental Health Continuum-Short Form (MHC-SF) in Setswanaspeaking South Africans. Clinical Psychology and Psychoterapy, 15, 181-192.

Keyes, C. L. M., Dhingra, S. S., \& Simoes, E. J. (2010). Change in level of positive mental health as a predictor of future risk of mental illness. American Journal of Public Health, 100(12), 2366-2371.

Keyes, C. L. M., Eisenberg, D., Perry, G. S., Dube, S. R., Kroenke, K., \& Dhingra, S. S. (2012). The relationship of level of positive mental health with current mental disorders in predicting suicidal behavior and academic impairment in college students. Journal of American College Health, 60(2), 126-133.

Keyes, C. L. M., \& Simoes, E. J. (2012.). To flourish or not: Positive mental health and all cause mortality. American Journal of American Public Health, 102(11), 2164-2172

Kłym, M., Karaś, D., Najderska, M., Cieciuch, J. (2014, July). Polish Version of the Questionnaire for Eudaimonic Well-Being (QEWB). Paper presented at the 28th International Congress of Applied Psychology, Paris, France.

Lamers, S. M. A., Westerhof, G. J., Bohlmeijer, E. T., ten Klooster, P. M., \& Keyes, C. L. M. (2011). Evaluating the psychometric properties of the Mental Health Continuum-Short Form (MHC-SF). Journal of Clinical Psychology, 67(1), 99-110. 
Running head: POLISH ADAPTATION OF MENTAL HEALTH CONTINUUM-SHORT FORM

Lamers, S. M., Glas, C. A., Westerhof, G. J., \& Bohlmeijer, E. T. (2012). Longitudinal evaluation of the mental health continuum-short form (MHC-SF). European Journal of Psychological Assessment, 28(4), 290-296.

Makowska, Z. \& Merecz, D. (2001). Polska adaptacja kwestionariuszy Ogólnego Stanu Zdrowia Davida Goldberga: GHQ-12 i GHQ-28 [Polish Adaptation of Goldberg's General Health Questionnaire: GHQ-12 and GHQ-28]. In: Z. Makowska \& D. Merecz (Eds.), Ocena zdrowia psychicznego na podstawie badań kwestionariuszami Davida Goldberga [The Assessment of Mental Health based on David Goldberg's Questionnaires] (pp. 191-264). Łódź; Instytut Medycyny Pracy im. Prof. J. Nofera. Marsh, H. W., Hau, K.-T. \& Wen, Z. (2004). In Search of Golden Rules: Comment on Hypothesis-Testing Approaches to Setting Cutoff Values for Fit Indexes and Dangers in Overgeneralizing Hu and Bentler's (1999) Findings. Structural Equation Modeling, $11(3), 320-341$.

Robitschek, C., \& Keyes, C. L. M. (2009). The structure of Keyes' model of mental health and the role of personal growth initiative as a parsimonious predictor. Journal of Counseling Psychology, 56(2), 321-329.

Ryan, R. M. \& Deci, E. L. (2001). On happiness and human potentials: A review of research on hedonic and eudaimonic well-being. Annual Review of Psychology, 52, 141-166.

Ryff, C. D. (1989). Happiness is everything, or is It? Explorations on the meaning of psychological well-being. Journal of Personality and Social Psychology, 57, 10691081.

Waterman, A. S., Schwartz, S. J., Zamboanga, B. L., Ravert, R. D., Williams, M. K., Agocha, V. B, Kim, S. Y., Donnellan, M. B. (2010). The Questionnaire for Eudaimonic WellBeing: Psychometric properties, demographic comparisons, and evidence of validity. The Journal of Positive Psychology, 5(1), 41-61. 
Watson, D., \& Clark, L.A. (1994). The PANAS-X: Manual for the Positive and Negative Affect Schedule - Expanded Form. Iowa Research Online. Retrieved: November, 7th, 2013 from:

http://ir.uiowa.edu/cgi/viewcontent.cgi?article=1011\&context=psychology_pubs

World Health Organization (1948). Constitution of the World Health Organization, retrieved November, 3rd, 2013, from:

http://www.who.int/governance/eb/who_constitution_en.pdf

World Health Organization (2004). Promoting mental health: Concepts, emerging evidence, practice - summary report. Geneva: World Health Organization. Retrieved November, 8th, 2013, from: http://www.who.int/mental_health/evidence/en/promoting_mhh.pdf 